\title{
Introduction Of Poloidally Separated Doppler Reflectometers In Experimental Advanced Superconducting (EAST) Tokamak
}

\author{
xiaohui zhang ${ }^{* \dagger}$ \\ University of Science and Technology of China, Hefei, Anhui 230026, China \\ E-mail: zxhuiamail.ustc.edu.co
}

\section{Another Author}

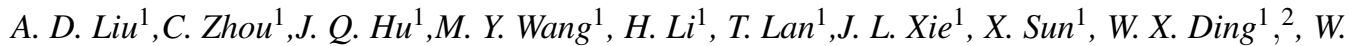 \\ D. $\mathrm{Liu}^{1}$, and C. X. Yu ${ }^{1}$, \\ ${ }^{1}$ University of Science and Technology of China, Hefei, Anhui 230026, China \\ ${ }^{2}$ University of California at Los Angeles, Los Angeles, CA 90095,USA \\ E-mail: Laddustc.edu.cD
}

\begin{abstract}
Two poloidally separated Doppler reflectometers have recently been set up In Experimental Advanced Superconducting (EAST) Tokamak.They contain two independent V-band $(50-75 \mathrm{GHz})$ systems which are designed for next EAST experimental campaign in the spring of 2015 year.The optical system are consist of two lens,some corrugated wave-guides and mirror combination. The mirror combination are consist of a steerable flat mirror and a fixed parabolic mirror which are optimized to improve the spectral resolution and adjust the launching angle. The incidence angle can cover the range $\pm 20^{\circ}$ by the steerable mirror enabling the wave number range $4-22 \mathrm{~cm}^{-1}$, with the wave number resolution around $2 \mathrm{~cm}^{-1}$.A synthesizer is used as the source and a $20 \mathrm{MHz}$ single band frequency modulator is used to get a differential frequency for heterodyne detection.This systems will be dedicated in investigation in zonal flow and Coherent Modes.Considering some indications of them have been found in last campaign. Poloidal mode number can be confirmed by correlation analysis and toroidal mode number can also be got through correlation analysis with 8 channel $D B S$ which is set up in another port. What is more; density fluctuation and radial electric field fluctuation can be detected at the same time.
\end{abstract}

First EPs Conference on Plasma Diagnostics - 1st ECPD,

14-17 April 2015

Villa Mondragone, Frascati (Rome) Italy

\footnotetext{
${ }^{*}$ Speaker.

${ }^{\dagger}$ Directed by A. D. Liu and C. X. Yu
} 
1. ...

\section{References}

[1] C. Zhou,A. D. Liu,X. H. Zhang,J. Q. Hu,M. Y. Wang, H. Li, T. Lan, J. L. Xie, X. Sun, W. X. Ding, W. D. Liu, and C. X. Yu, Microwave Doppler reflectometer system in the Experimental Advanced Superconducting Tokamak, REVIEW OF SCIENTIFIC INSTRUMENTS 84103511 (2013).

[2] book B. Baggins, There and back again, Imladris Editions, Rivendell 3018. 